\title{
Evaluation of the Effects of Intravenous and Percutaneous Low Level Laser Therapy in the Management of Shoulder Myofascial Pain Syndrome
}

\author{
Sirous Momenzadeh ${ }^{1,2}$, Vahid Akhyani ${ }^{3 *}$, Zahra Razaghi ${ }^{1}$, Asghar Ebadifar $^{4}$, Mohammadzaki Abbasi ${ }^{5}$ \\ ${ }^{1}$ Laser Application in Medical Sciences Research Center, Shahid Beheshti University of Medical Sciences, Tehran, Iran \\ ${ }^{2}$ Department of Anesthesiology and Pain Medicine, Imam Hossein Hospital, Shahid Beheshti University of Medical \\ Sciences, Tehran, Iran \\ ${ }^{3}$ Department of Anesthesiology and Pain Medicine, Akhtar Hospital, Shahid Beheshti University of Medical Sciences, \\ Tehran, Iran \\ ${ }^{4}$ Dentofacial Deformities Research Center Orthodontics Department, Shahid Beheshti University of Medical Sciences, \\ Tehran, Iran \\ ${ }^{5}$ Department of Urology, Boushehr University of Medical Sciences, Boushehr, Iran
}

\section{*Correspondence to VahidAkhyani, MD; Department of Anesthesiology and Pain Medicine, Akhtar Hospital, Shahid Beheshti University of Medical Sciences, Tehran, Iran. Tel: +98-2122612252; Fax: +98-2122612252; \\ Email: vahid_akhyani@yahoo.com}

Published online 7 January 2016

\begin{abstract}
Introduction: Myofascial pain syndrome (MPS) treatment is challenging with a high recurrence rate and still lacks a clear treatment frame. Therefore research on new, more efficient and long lasting effect treatment modalities is necessary. This study looked at the effects of intravenous laser therapy (IVL) and percutaneous low level laser (PLLL) in the management of shoulder MPS.

Methods: In this randomized controlled trial, 30 patients fulfilling inclusion criteria were randomly equally allocated to 3 groups, control, IVL and PLLL. Control group received 12 sessions of placebo low level laser, IVL group received 12 sessions of IVL therapy, and PLLL group received 12 sessions of PLLL therapy. All patients were trained for better body posture, body mechanics, gentle massage of trigger points, stretching exercises of affected muscle (trapezius), and received $10 \mathrm{mg}$ of oral nortriptyline regimen every night for 3 months. Outcomes included pain severity, functional disability, and quality of life. Patients were assessed using Numeric Rating Scale (NRS), Pain Disability Index (PDI), and Short Form Health Survey (SF-12). Data collected were analyzed using analysis of variance (ANOVA), Mann-Whitney and $t$ tests.

Results: The mean of PDI and maximum pain intensity during day and night significantly reduced in both PLLL and IVL groups compared to control group. Although pain severity and PDI reduction was more pronounced in IVL group compared to PLLL group, the differences were not statistically significant. Also, quality of life statistically significantly improved in both IVL and PLLL groups compared to control group was more, and although higher in IVL group, the difference was not statistically significant when compared to PLLL group. No side effects were observed in the intervention groups.

Conclusion: Intravenous laser and PLLL therapy had a positive effect on pain severity and PDI reduction, and quality of life in this study. Also no adverse event was recorded. Thus, intravenous lasers and PLLL therapy seem to be effective complementary modalities in managing patients with shoulder MPS.
\end{abstract}

Keywords: Laser therapy; LLLT; IVL; Myofascial pain syndrome. life span and general health due to technological progress in medical fields lead to increased overall years of work. In addition progress in information technology (IT) is profoundly changing the pattern of work in many fields, with much more time spent behind desks and computers. These long hours of sitting are stresses which can help in development of chronic pains. Therefore we can expect

Please cite this article as follows: Momenzadeh S, Akhyani V, Razaghi Z, Ebadifar A, Abbasi M. Evaluation of the effects of intravenous and percutaneous low level laser therapy in the management of shoulder myofascial pain syndrome. / Lasers Med Sci. 2016;7(1):16-20. doi:10.15171/jlms.2016.04. 
myofascial pain to grow in prevalence as both sedentary life which increases progressively and technologic progress provide work conditions favorable for development of this condition.

Despite decades of research on MPS, this disorder still lacks a clear treatment frame ${ }^{4}$ and MPS management is based on multimodal approach. ${ }^{5}$ Actual treatment modalities comprise awareness of associated factors, medication, stretching exercises, acupuncture, injections, manual therapy, ultrasound, laser therapy, electrical stimulation, transcutaneous electrical nerve stimulation and biofeedback, ${ }^{2,6}$ but no single combination has provided us with an effective and efficient treatment method since recurrence is still high. ${ }^{7}$ Many studies have been performed on the effects of percutaneous low level laser (PLLL) on MPS, and showed some positive results, ${ }^{5}$ but many of these studies lack validity. Although laser protocols in terms of duration, dosage and wavelength have yet to be determined; Low level laser therapy (LLLT) can be introduced as an adjunctive modality for treatment of chronic pain conditions. ${ }^{4}$

Intravenous laser therapy (IVL) has been applications in many different areas of medicine with a growing interest. IVL has laser specific analgesic, spasmolytic and sedative effects among many other. ${ }^{8}$ Many theories exist on the mechanisms of effect of this relatively new modality. But beside all the laser related effects seen in LLLT, an additional anticipated action of intravenous laser blood therapy on MPS can be based on a number of its effects listed by Weber in "The Intravenous laser blood irradiation-Introduction of a new therapy": Stimulation of the NO-production in monocytes with vasodilatation and improvement of endothelial dysfunction; Fusion of mitochondria to "giant mitochondria" with increase of ATP-production in the respiratory chain; Improvement of regeneration of erythrocytes and of microcirculation; Normalization of tissue metabolism by improvement of hypoxia; Normalization of the cell membrane potential; Improvement of the anti-oxidant enzymatic system with antitoxic effect. ${ }^{8}$ IVL is a new treatment method which has been proposed for management of chronic disorders as well as chronic pain syndromes. Based on the mechanisms of action of this modality, and its effects on inflammation, microcirculation and biostimulation as a whole, ${ }^{8}$ we can anticipate it to be effective in management of MPS. In addition we can expect some advantages of IVL compared to LLLT considering its delivery mode which helps ensure a more direct and general effect while reducing the energy dosage to achieve the same effects.

Furthermore most investigations on intravenous lasers and their various effects as well as LLLT on MPS have been performed mainly by Russian researchers. Unfortunately most of these studies are not available for non-Russian speaking scholars since few of them have been translated in English. So by performing this study we help building a knowledge background for these technologies, their indications and the procedures related to their use.

As stated by Argoff et $\mathrm{al}^{9}$ social, psychological and behav- ioral disturbances may precede or follow the development of pain in MPS. Patients may present with different psychological symptoms such as anger, depression, anxiety and frustration. Few studies have assessed psychological factors in MPS. ${ }^{9}$ In addition, very limited studies have worked on the functional assessment of MPS. In this study, quality of life was assessed using The Short Form Health Survey (SF-12) and disability was assessed using Pain Disability Index (PDI).

Research on new, more efficient and long lasting effect treatment modalities for MPS is necessary. In this study we intended to evaluate the effects of IVL in the management of shoulder MPS in comparison to PLLL.

\section{Methods}

This study was designed as a prospective randomized controlled clinical trial. After approval from the ethic committee of Shahid Beheshti University of Medical Sciences, 30 patients suffering from MPS that referred to pain clinics of 4 different hospitals (Shohaday-e-Tajrish hospital, Akhtar hospital, Labafinejad hospital and Emam Hossein hospital) were enrolled. Diagnosis of shoulder MPS was confirmed by two pain specialists as well as by a physical medicine and rehabilitation specialist separately for the patients to be included. These three specialists had previously undergone at least 3 hours of training for diagnosis of MPS. After full disclosure in regard to this study, all patients enrolled signed an informed consent form. To ensure maximum blinding, all laser treatments were performed by a pain specialist while all assessments and data collection of before, during and after treatment were achieved by the other pain specialist. Data analysis was executed by a third person (analyst) with the assessor and analyst also unaware of the type of modality being applied to each patient.

\section{Subjects}

Patients included all fulfilled at least 3 MPS criteria. Diagnosis was made based on the following criteria:

- Pain: chronic pain of shoulder soft tissues (myofascial) that affects a focal or regional portion of the body

- $\quad$ Myofascial trigger point: A tender spot (trigger point) in an affected muscle (trapezius muscle)

- Referral Pain: Referral of pain to a zone of reference. All patients suffering from shoulder MPS referring to pain clinics of Shohaday-e-Tajrish hospital, Akhtar hospital, Labafinejad hospital and Emam Hossein hospital in 2013 constituted the study population.

\section{Inclusion Criteria}

- $\quad$ Age between 18 to 60

- Suffering from shoulder MPS with at least one trigger point

- Patients should have been affected by shoulder MPS for at least 3 months

- $\quad$ No previous IVL or LLLT

- Being at least 1 month without any other treatment 
modality, such as NSAIDS, corticosteroids, etc

- Normal neurologic examination (deep tendon reflexes, manual muscle testing and sensory examination)

- No contraindication for use of nortriptyline

- No other disease explaining their symptoms (rheumatoid arthritis or fibromyalgia or ...)

- Complete informed agreement of patients to participate in the study.

\section{Exclusion Criteria}

- Patients who had stage 3 and 4 osteoarthritis, infectious disease, a tumor, scoliosis, pregnancy, chronic obstructive lung disease and bleeding diathesis were excluded

- Patients with history of accident and severe trauma

- Willingness to receive other treatment options

- Patients suffering from acute or chronic systemic diseases (cardiovascular, hepatic and renal diseases, ...)

- Cases of uncontrolled hypertension, psychotic disorders

- Presence of diseases such as radiculopathy, neuropathy and myelopathy

The sampling method was simple sampling. To assure allocation concealment randomization and group assignment was conducted by an independent observer. The sample subjects were randomly assigned to 3 groups of at least 10 patients using randomization software (Microsoft Excel 2010).

\section{Procedures}

The first group (control) were 10 patients who received SHAM laser; the second group (IVL) were 10 patients who received IVL laser; the third group (PLLL) were 10 patients who received PLLL as treatments. All patients were trained by an experienced physical therapist for better body posture, body mechanics, gentle massage of the Trigger points, stretching exercises of the affected muscle (trapezius), and received oral nortriptyline regimen. Nortriptyline regimen was of $10 \mathrm{mg}$ nortriptyline every night for 3 months.

The IVL group received one session of 20 minutes intravenous laser blood irradiationevery two days except Fridays during 4 weeks for a total of 12 sessions. IVL was applied on the most appropriate and largest vein of the upper extremities through disposable $22 \mathrm{G}$ intravenous catheter and one-use optical fiber needle. Laser device used for IVL was Helium-Neon (He-Ne) Laser $632.8 \mathrm{~nm}$ IV 2 mw continuous wave. (He-Ne Laser multi-function cure instrument, model KX-350-1B, Guillin Kangxing Medical Instrument Co. Ltd. China).

The low level laser group received one session every two days except Fridays for a total of 12 sessions. LLLT was applied transcutaneously at 1 point over the pain area (center of trigger point) in a contact and fixed mode with an energy density of $20.35 \mathrm{~J} / \mathrm{cm}^{2}$ in 300 seconds for every trigger point (almost in $3 \mathrm{~cm}$ depth). Laser device used for LLLT was gallium aluminum arsenide (GaALAs) laser $810 \mathrm{~nm} 60 \mathrm{mw}$ continuous wave, $0.2211 \mathrm{Cm} 2$ Spot Area,
$20.35 \mathrm{~J} / \mathrm{cm}^{2}$ per point. (GaALAs semiconductor laser cure instrument, model KX-IR-2A, Guilin Kangxing Medical Instrument Co. Ltd. China).

The Low level laser placebo group had the probe not turned on and applied as for the LLLT group. Placebo laser sessions were every two days except Fridays for a total of 12 sessions.

\section{Data Collection and Validation}

For every patient enrolled, physical examination was performed; Pain Disability Index (PDI), Numeric Rating Scale (NRS), The Short Form Health Survey (SF-12) were filled before treatment started. NRS assessed maximum of pain intensity at night, during rest and activity.

At the end of the 12 treatment sessions, NRS, PDI, SF-12 were filled and as well as at 1 and 3 months after last treatment session for follow up.

\section{Statistical Tests}

Statistical analysis was carried out using SPSS version 16 (SPSS Inc., Chicago, IL). Data collected were analyzed using analysis of variance (ANOVA), Mann-Whitney and $t$ tests.

\section{Results}

Thirty patients participated in this study which 21 (70\%) were male and $9(30 \%)$ were female. Ten patients were treated with IVL, of which 2 (20\%) were male and 8 (80\%) were female; 10 patients were treated with PLLL, of which 3 (30\%) were male and 7 (70\%) were female; and 10 were treated with laser placebo, of which $4(40 \%)$ were male and $6(60 \%)$ were female.

Patients' demographic characteristics including age are listed in Table 1 . The mean of maximum severity of pain during day and night (mean of maximum pain at sleep, during activity and at rest), the mean of PDI, and the mean score of World Health Organization-Quality of Life questionnaire (SF-12) for the study period are presented in Figures 1-3.

All parameters, age, sex, mean of pain intensity during day and night, and mean of PDI matched in the 3 groups at the beginning of study, and no significant statistical difference was observed between them.

In this study the mean of maximum pain intensity during day and night at the end of treatment and follow-up sessions 1 and 3 months after treatment showed significantly $(P<0.0001)$ more reduction in both IVL and PLLL groups compared to placebo laser group, but although the reduction of pain severity was more pronounced in IVL group compared to PLLL group, it was not statistically signifi-

Table 1. The Mean and Standard Deviation of Age of the Participants in the Studied Groups

\begin{tabular}{lccc}
\hline Treatment Group & Min. & Max. & Mean \pm SD \\
\hline IVL & 22 & 61 & $44.70 \pm 12.59$ \\
PLLL & 33 & 72 & $45.20 \pm 12.76$ \\
PLT & 24 & 75 & $47.40 \pm 17.91$ \\
\hline Ab
\end{tabular}

Abbreviations: IVL, intravenous laser therapy; PLLL, percutaneous low level laser; PLT, placebo low level laser therapy. 


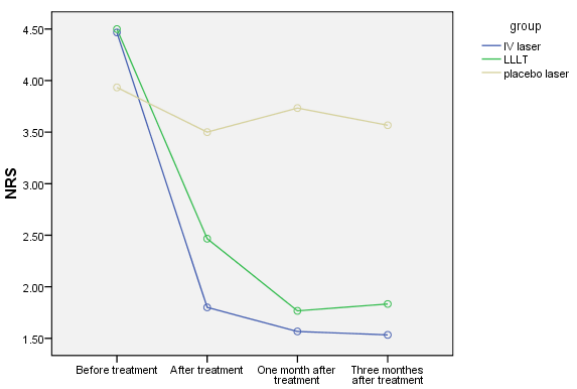

Figure 1. Mean of Maximum Pain Intensity in Patients During Day and Night (the Mean of Maximum Pain During Activity and Rest and Sleep) in the Studied Groups.

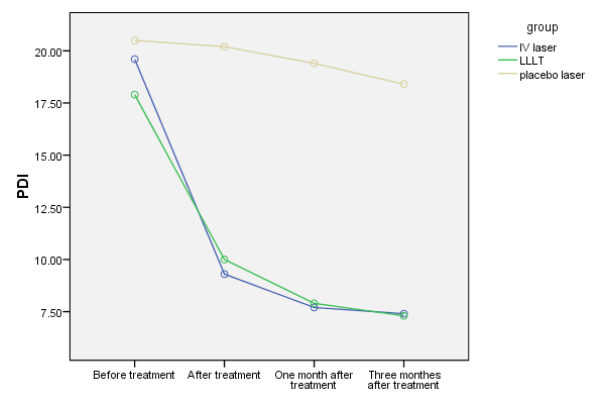

Figure 2. Mean of Pain Disability Index (PDI) of Patients in the Studied Groups.

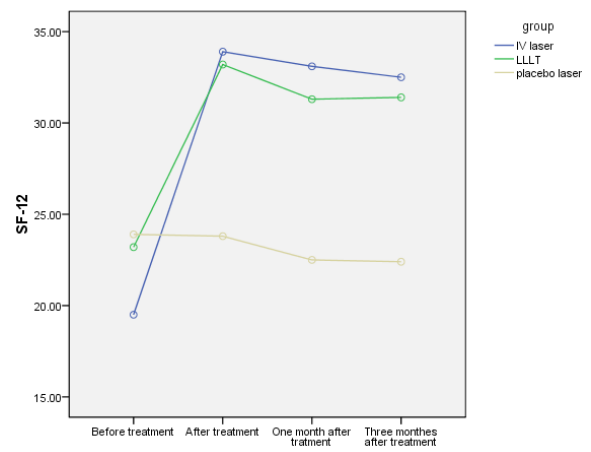

Figure 3. Mean Scores of Short Form Quality of life World Health Organization Questionnaire (SF-12) of Patients in the Study Period.

cant $(P=0.2)$

Similar results were obtained for PDI, where our findings showed that the mean of PDI at the end of treatment and follow-up sessions 1 and 3 months after the end of treatment also had significantly $(P<0.0001)$ more reduction in both IVL and PLLL groups compared to placebo laser group, with more reduction in IVL group compared to PLLL group, although the difference was not statistically significant $(P=0.9)$.

Also according to our results, the score from the short form quality of life World Health Organization (SF-12) questionnaire improved from low quality to high quality of life and stayed constant in the high quality range until the end of the follow up period in both IVL and LLLT groups, while when compared to the placebo laser group these improvement in quality of life observed in both IVL and PLLL groups were statistically significantly increased $(P<0.0001)$. Although the improvement of the quality of life in patients treated with IVL was higher than with PLLL, the difference between the two groups was not significant $(P=0.9)$.

No side effects or adverse event were observed or reported in the intervention groups during the course of this study.

\section{Discussion}

We evaluated the effects and safety of IVL and (PLLL) therapy in the management of shoulder MPS.

Our findings showed that the mean of maximum intensity of pain in patients during day and night during the study period decreased more in IVL and PLLL groups and pain was significantly improved compared to the control group $(P<0.0001)$, also although this decrease was higher in the IVL group compared to the PLLL group, the difference was not statistically significant $(P=0.2)$.

Similarly, the reduction of PDI of our patients in both IVL and PLLL groups was significantly more pronounced when compared to the placebo group $(P<0.0001)$. Here also, although there was a higher decrease in the IVL group compared to the PLLL group, the difference was not statistically significant $(P=0.9)$. These results demonstrate the positive effects of IVL and PLLL when compared to PLT in the management of pain of patients with shoulder MPS. However, this study was unable to demonstrate any difference in efficacy between IVL and PLLL, as despite a slight better effect of IVL, there was no significant superiority to PLLL in the treatment of pain of these patients.

In previous studies also, LLLT was reported to be effective in the treatment of MPS as an adjunctive treatment. ${ }^{4,5}$

In Gur et al study, the use of LLLT was effective in treating neck MPS compared to placebo. ${ }^{10}$ Carrasco et al also reported that LLLT was effective in pain reduction of patients with MPS. ${ }^{11}$

In regard to improvements in quality of life, in our study we observed that patients treated with IVL and PLLL had more significant $(P<0.0001)$ improvement in quality of their lives compared to the control group as well as when compared to before treatment. Here again, there was no statistical difference $(P=0.9)$ in improvement of quality of life between the IVL group and the PLLL group, despite a better quality of life in the IVL group when compared to the PLLL group. These results showed positive effects of IVL and PLLL when compared to PLT in improving the quality of life of patients with shoulder MPS. This study failed to demonstrate any superiority of IVL over PLLL in improving the quality of life of these patients.

Gür et al in another study on the effects of low dose amitriptyline and LLLT on quality of life and clinical symptoms in fibromyalgia, suggested that both laser therapies and amitriptyline are effective on quality of life and clinical symptoms in fibromyalgia. ${ }^{12}$ Also Bjordal et al explained in their paper, LLLT reduces disability and pain both in the short and medium term. ${ }^{13}$ 
In addition, considering the fact that none of our patients in the study period experienced any side effects or adverse event, IVL or PLLL can at least can be considered at least safe methods in the treatment of shoulder MPS.

Overall, our findings in this study, like previous studies, support that MPS still lacks a clear treatment frame ${ }^{4}$ and its management should be based on a multimodal approach. $^{5}$

\section{Conclusion}

In regard to the findings of our study that indicate positive effects of the use of IVL or PLLL in combination with nortriptyline and patients' physical therapy education to the patients, in reducing pain and PDI as well as improving quality of life of patients with shoulder MPS, and considering the fact that no patients experienced side effects or adverse events, IVL and PLLL seem to be effective and can be considered as complementary modalities in the management of shoulder MPS. Despite better results observed with IVL in comparison to PLLL in all variables studied, our study failed to demonstrate any statistically significant superiority of IVL over PLLL, which may be due to the volume of our sample. Therefore, in order to achieve more accurate and definite results in the use of IVL in MPS, we recommend similar studies with more subjects.

\section{Conflict of Interest}

The author has no conflict of interest to declare.

\section{References}

1. Rickards LD. The effectiveness of non-invasive treatments for active myofascialtrigger point pain: a systematic review of the literature. Int J Osteopath Med. 2006;9(4):120-136. doi:10.1016/j.ijosm.2006.07.007.

2. Furlan AD, Yazdi F, Tsertsvadze A, et al. A systematic review and meta-analysis of efficacy, cost-effectiveness, and safety of selected complementary and alternative medicine for neck and low-back pain. Evid Based Complement Altern Med. 2012;2012:953139. doi:10.1155/2012/953139.
3. Çeliker R, Atalay A, Guven Z. Health-related quality of life in patients with myofascial pain syndrome. Curr Pain Headache Rep. 2010;14(5):361-366. doi:10.1007/s11916010-0141-7.

4. Annaswamy TM, De Luigi AJ, O’Neill BJ, Keole N, Berbrayer D. Emerging concepts in the treatment of myofascial pain: a review of medications, modalities, and needle-based interventions. PM R. 2011;3(10):940-961. doi:10.1016/j.pmrj.2011.06.013.

5. Ilbuldu E, Cakmak A, Disci R, Aydin R. Comparison of laser, dry needling, and placebo laser treatments in myofascial pain syndrome. Photomed Laser Surg. 2004; 22(4):306-311.

6. Rayegani SM, Bahrami MH, Samadi B, Sedighpour L, Mokhtarirad MR, Eliaspour D. Comparison of the effects of low energy laser and ultrasound in treatment of shoulder myofascial pain syndrome: a randomized single-blinded clinical trial. Eur J Phys Rehabil Med. 2011;47(3):381-389.

7. Yap EC. Myofascial pain- an overview. Ann Acad Med Singapore. 2007;36:43-8.

8. Weber MH. The intravenous laser blood irradiationintroduction of a new therapy. Ger J Acupuncture Relat Tech. 2007;50(3):12-23.

9. Argoff C, Smith H, Evans RW. Myofascial pain syndrome. http://www.medmerits.com/index.php/article/ myofascial_pain_syndrome.

10. Gur A, Sarac AJ, Cevik R, Altindag O, Sarac S. Efficacy of $904 \mathrm{~nm}$ gallium arsenide low level laser therapy in the management of chronic myofascial pain in the neck: a double-blind and randomize-controlled trial. Lasers Surg Med. 2004;35(3):229-235.

11. Carrasco TG, Guerisoli LD, Guerisoli DM, Mazzetto MO. Evaluation of low intensity laser therapy in myofascial pain syndrome. Cranio. 2009;27(4):243-247.

12. Gür A, Karakoc M, Nas K, Cevik R, Sarac J, Ataoglu S. Effects of low power laser and low dose amitriptyline therapy on clinical symptoms and quality of life in fibromyalgia: a single-blind, placebo-controlled trial. Rheumatol Int. 2002 Sep;22(5):188-93. Epub 2002 Jul 6.

13. Bjordal JM, Lopes-Martins R, Johnson MI, Chow R. Inaccuracies in laser therapy meta-analysis for neck pain? J Physiother. 2010; 56(4):282. 